\title{
La situación de discapacidad y su relación con la accesibilidad universal en la atención primaria de salud, comuna de Rancagua ${ }^{1-2}$
}

\author{
Carmen Nadal Agost ${ }^{3}$ \\ Marcelo Torres Fuentes ${ }^{4}$
}

\section{RESUMEN}

Chile en el año 2008 ratifica la Convención sobre los Derechos de las Personas con Discapacidad de las Naciones Unidas del año 2006, para, posteriormente, proclamar en el 2010 la ley $\mathrm{N}^{\circ} 20.422$ que establece normas sobre igualdad de oportunidades e inclusión social, asegurando el disfrute de sus derechos y la eliminación de cualquier forma de discriminación. Esta ley inspiró una política pública implementada a través del Plan Nacional de Discapacidad 2013-2020 entre cuyas temáticas se encuentra la accesibilidad universal. Es en ese contexto que el equipo de Salud del CESFAM No6 de Rancagua instaló, dentro de su agenda pública, la temática de discapacidad con la intención de visibilizar a este grupo de la población en la construcción de políticas locales inclusivas. Para el logro de lo anterior, se realizó una investigación mixta cuyo objetivo fue conocer en profundidad la visión respecto de las principales barreras y fortalezas en la accesibilidad de las personas en situación de discapacidad.

Palabras clave: Política Pública - Accesibilidad Universal - Discapacidad Derechos Humanos.

\section{A situação da deficiência física e sua relação com a acessibilidade universal na atenção primária à saúde na comuna de Rancagua.}

\section{RESUMO}

Chile no ano 2008, ratificou a Convenção sobre os Direitos das Pessoas com Deficiência das Nações Unidas do ano 2006, para posteriormente

1 Artículo recibido el 30/03/2017. Artículo aprobado el 01/08/2017

2 El contexto institucional de esta investigación es el Centro de Salud Familiar №6 Ignacio Caroca de la Corporación Municipal de Rancagua.

3 Chilena, Médico Familiar, directora del Centro de Salud Familiar N ${ }^{\circ} 6$ Ignacio Caroca C. en Rancagua, Chile, e-mail: cnadal@saludcormun.cl

4 Chileno. Trabajador Social. Investigador y Académico Escuela de Trabajo Social Universidad Católica Silva Henríquez y Universidad Tecnológica de Chile, e-mail: mtorresf@saludcormun.cl 
La situación de discapacidad y su relación con la accesibilidad universal en la atención primaria de salud, comuna de Rancagua / Nadal - Torres

proclamar no 2010 a Lei $n^{\circ} 20.422$, que estabelece regras sobre igualdade de oportunidades e inclusão social, garantindo o deleite de seus direitos e eliminação de qualquer forma de discriminação. Esta lei inspirou uma política pública implementada através do Plano Nacional de Incapacidade 2013-2020, cujo tema é a acessibilidade universal. É neste contexto que a Equipe de Saúde do CESFAM No 6 de Rancagua instalou, dentro de sua agenda pública, o tema da deficiência física com a intenção de tornar visível este grupo da população na construção de políticas locais inclusivas. Para o logro do anterior, realizou-se uma pesquisa mista, cujo objetivo foi conhecer em profundidade a visão sobre as principais barreiras e fortalezas na acessibilidade das pessoas em situação com deficiência física.

Palavras-chave: Política Pública - Acessibilidade Universal - Deficiência Física - Direitos Humanos

\section{The situation of disability and its relation with universal access to primary health care in the Municipality of Rancagua}

\section{ABSTRACT}

In 2008, Chile ratified the 2006 United Nations' Convention on the Rights of Persons with Disabilities and later, in 2010, proclaimed the Law No. 20,422, establishing norms on opportunity equality and social inclusion, ensuring their rights and eliminating any form of discrimination. This law inspired a public policy implemented through the National Plan for Disability 20132020 which includes, among others, universal access. In this context, the Health working team of CESFAM N6 at Rancagua included disability in its public agenda with the purpose of making this population group visible in developing inclusive local policies. To that end, there was a mixed research aimed to get feedback regarding the main barriers and strengths in the access of persons with disability. It was applied with very limited resources and there is a lot more to be done to support the education of children with disabilities. In this context, the Health professionals who work in CESFAM N6 of Rancagua incorporated the disability issue in their agenda in order to include that population in local policies. A mixed research was done to achieve the perception of barriers and strengths in accessibility of people with disabilities.

Keywords: Public policy - universal accessibility - disability - human rights -health 


\section{Presentación}

En una sociedad globalizada como la del siglo XXI, emergen nuevos desafíos para lograr una adecuada convivencia colectiva que incluya la diversidad física y social propia de los seres humanos. Surge entonces, una definición de "discapacidad" impulsada por la mirada biopsicosocial, que señala que la deficiencia no radica en la persona discapacitada, sino más bien, en las condiciones que su medio no le provee para poder desarrollar su proyecto de vida.

Basado en la Declaración Universal de los Derechos Humanos (1948), se pretende garantizar, por los Estados Miembros de las Naciones Unidas, la eliminación de barreras hacia las personas en situación de discapacidad (PsD) a través de la Convención Internacional sobre los Derechos de las Personas con Discapacidad declarada en el año 2006. Dicha Convención fue ratificada por el Estado Chileno y actuó como fuente de inspiración para implementar una política pública que abordara las problemáticas en relación a la discapacidad en el país y la ley No 20.422 que "Establece Normas sobre la Igualdad de Oportunidades e Inclusión Social de Personas con Discapacidad", considerando dimensiones relevantes como PsD, accesibilidad universal, diseño universal, políticas públicas, derechos humanos y vulnerabilidad.

En el año 2005, se realizó por primera vez en Chile una encuesta nacional a cargo del Fondo Nacional de la Discapacidad (FONADIS), cuyos objetivos fueron conocer la prevalencia de discapacidad en Chile y caracterizar a este tipo de población. Como la Organización Mundial de la Salud (OMS) precisó que los estudios en discapacidad poseen una vigencia de 10 años, se publicó en el año 2015 la segunda versión de esta encuesta, cuya finalidad fue aportar un insumo clave para la formulación de políticas públicas con mayor pertinencia en materia de inclusión social para las personas en situación de discapacidad, pues se abordó la temática desde una mirada integral, incorporando un análisis de la persona en relación con su ambiente, su vida personal, su condición de 
salud y el desempeño logrado en distintos ámbitos de la sociedad (II Estudio Nacional de la Discapacidad, 2015).

Según lo señalado por la (OMS) el crecimiento de la población, las condiciones de trabajo, los tipos de alimentos que se consumen, el ritmo de vida y el proceso de envejecimiento, serían las causas que han aumentado la cifra de personas en situación de discapacidad. Mientras, a nivel mundial, se registran cerca de 650 millones de PsD (equivalentes al 10\% del total de población), en Chile se registran 2.836.818 que representan al 16,7\% del país (II Estudio Nacional de la Discapacidad, 2015).

En el país, 1 de cada 8 personas vive con algún grado de discapacidad, siendo el 33,67\% de tipo leve (dificultad para llevar a cabo actividades de la vida diaria), el 21,23\% de tipo moderado (disminución de su capacidad para realizar la mayoría de las actividades cotidianas) y el $8.32 \%$ del tipo severo (imposibilidad de realizar sus actividades cotidianas).

Actualmente, se puede señalar que las personas que están ocupadas en el país son 7.680.211, el total de personas desocupadas es 527.338 y el total de personas que se encuentran inactivas, de 3.229.411. En este ámbito, y en relación a las PsD, las personas ocupadas representan el 13,3\%, mientras, las desocupadas el $14,9 \%$ y las inactivas, el 31,6\%. En base a esta información se puede afirmar que 1 de cada 10 personas que trabaja, está en situación de discapacidad; a su vez, 1 de cada 10 personas que está buscando trabajo, se encuentra en esa misma situación. Por último, 3 de cada 10 personas que no participa del mercado laboral, son personas en situación de discapacidad (II Estudio Nacional de la Discapacidad, 2015).

Desde la dimensión educacional se señala que el promedio de años de estudio para las personas que están en situación de discapacidad es de 8,6 años, mientras que para las personas que no están bajo esa situación, es de 11,6 años. Esto evidencia que las personas en situación de discapacidad estudian en promedio menos años que la población nacional (11 años). Cuando se obser- 
va la población en situación de discapacidad según severidad, se evidencia que aquella más severa estudia en promedio 7,1 años, mientras que las que están en leve o moderada, estudian en promedio 9,6 años (II Estudio Nacional de la Discapacidad, 2015).

Respecto a la autopercepción del estado de salud, hay una fuerte relación con la situación de discapacidad. Las PsD definen su salud como "mala o muy mala" en un $30,1 \%$ en comparación con las personas sin discapacidad, que sólo ascienden a un 2,3\% con esa misma respuesta. $\mathrm{Al}$ analizar este resultado, considerando los grados de severidad de la situación de discapacidad, la diferencia se hace más notoria (un 18,5\% del total de personas en situación de discapacidad leve a moderada, califica su salud como "mala o muy mala"; y un 46,4\% del total de personas en situación de discapacidad, como severa). Respecto a aquellas personas que definen su estado de salud como "bueno o muy bueno": un 70,6\% de las personas sin situación de discapacidad se califica dentro de estas categorías, un $22 \%$ en el grupo de personas en situación de discapacidad leve a moderada y en el grupo de personas en situación de discapacidad severa, solo un 9,8\%" (II Estudio Nacional de la Discapacidad, 2015, p. 133). Por último, la población adulta en situación de discapacidad y dependencia funcional corresponde a un 40,4\% del total, lo cual significa que 1.052 .787 personas adultas se encuentran en situación de discapacidad y además están en situación de dependencia (II Estudio Nacional de la Discapacidad, 2015).

Los antecedentes entregados por el diagnóstico comunal de la Oficina de Capacidades Diferentes de Rancagua (año 2015), señalan que la Región de O’Higgins supera la media nacional en un 2,2\% en relación a la cantidad de personas en situación de discapacidad. Su capital, la ciudad de Rancagua, registra 4.253 PsD y de ellas el 33,9\% se concentra en el sector oriente, específicamente el CESFAM 6 atiende a 452 PsD.

Además, se señala que los CESFAM 1, 4 y 6 son los que cuentan con todos los estándares necesarios para ser inclusivos al momento de 
entregar la atención médica. Sin embargo, es necesario conocer y abordar otros ámbitos asociados a la accesibilidad, entre los cuales destacan: el traslado hacia el centro de salud y el acceso a la información. Por ello, el equipo técnico del Centro de Salud Familiar nº de la comuna de Rancagua, desarrolló una investigación para medir la accesibilidad percibida por los usuarios y sus cuidadores.

\section{Desarrollo argumentativo del estudio}

A partir del 10 de diciembre del año 1948, la convivencia de la especie humana daría un paso trascendental. El poder bélico, político y económico revelado en las dos guerras mundiales de mediados del siglo XX, situó en grave peligro la subsistencia de la humanidad. Tras aquella devastadora experiencia, la Asamblea General de la Organización de Naciones Unidas (ONU), fijó la piedra angular para exigir la protección de las vidas humanas al proclamar los Derechos Humanos Universales que regirían la nueva relación entre los ciudadanos con su Estado. Estos derechos están definidos como las "garantías esenciales para que podamos vivir como seres humanos. Sin ellos no podemos cultivar ni ejercer plenamente nuestras cualidades, nuestra inteligencia, talento y espiritualidad" (Universidad de Salamanca, Naciones Unidas, Portal de Derechos Humanos). Además, los Derechos Humanos comparten ciertos rasgos característicos que los distinguen, ya que son: universales, innatos, irrenunciables, obligatorios, inalienables, imprescriptibles, indivisibles, inviolables y progresivos (Características de los Derechos Humanos, 2008). En la evolución histórica de estas garantías y luego de un largo proceso de trabajo entre los Estados Miembros de la ONU, Instituciones de Derechos Humanos y Organizaciones no gubernamentales, el 13 de diciembre del año 2006 se aprueba la Convención Internacional sobre los Derechos de las Personas con Discapacidad, cuyo principal objetivo radicó en trasladar los derechos reconocidos internacionalmente a ámbitos concretos que permitan identificar los medios para eliminar las barreras que enfrentan las personas con discapacidad, y lograr así el reconocimiento integral y el pleno ejercicio de esos 
derechos, en igualdad de oportunidades en las distintas esferas de la vida en sociedad (Guasch y Hernández, 2010). Proteger la vida humana, como objetivo principal de la Declaración Universal de los Derechos Humanos, es un imperativo moral, pues el ser humano, sujeto a un cuerpo, es visto e interpretado por la hermenéutica contemporánea como vulnerable, en el sentido de que puede ser dañado, a saber, vivir supeditado a riesgos y amenazas contra su integridad (Valdés, 2013).

Es así como la Convención sobre los Derechos Humanos de las Personas con Discapacidad incluye artículos cuya principal función es alertar sobre grupos o situaciones de vulnerabilidad, las cuales exigen contar con medidas de protección específicas, debido a que las personas en esta situación se encuentran en desventaja, y como consecuencia de ello, se ven afectados por una doble o múltiple discriminación, razón por la cual la Convención acuerda los siguientes principios: El respeto de la dignidad inherente al ser humano, la autonomía individual (incluida la libertad de tomar las propias decisiones, y la independencia de las personas); la no discriminación; la participación e inclusión plenas y efectivas en la sociedad; el respeto por la diferencia y la aceptación de las personas con discapacidad como parte de la diversidad y la condición humana; la igualdad de oportunidades; la accesibilidad; la igualdad entre el hombre y la mujer; el respeto a la evolución de las facultades de los niños y las niñas con discapacidad y de su derecho a preservar su identidad (Convención Internacional sobre los Derechos de las Personas con Discapacidad (CIPD), 2006).

Estos principios rigen hasta hoy para las personas portadoras o que viven una situación de alguna "discapacidad", cuya definición tuvo una evolución desde el tradicional modelo biomédico hacia el modelo social presentado por primera vez en el año 1976 por la organización inglesa de Unión de Personas con Discapacidad Física contra la Segregación (UPIAS). Dicho organismo la definía como una desventaja o limitación en la actividad causada por una organización y estructura social que tiene una escasa consideración por las personas con deficiencias, y por tanto, las excluye de la corriente 
principal de las actividades sociales (PLANDISC, 2013). En el año 2001, durante la 54 Asamblea Mundial de la Salud, la discapacidad fue considerada, en términos globales, como una "interacción multidireccional entre la persona y el contexto socio-ambiental" (Samaniego, 2006, p. 23). En otras palabras, las personas con déficit físico, sensorial o mental se encuentran en situación de discapacidad debido a la falta de oportunidades en su medio social, lo que se traduce en un mayor grado de vulnerabilidad. Estas perspectivas sociales se revalidan en el año 2006 con la definición de "discapacidad" declarada en la Convención Internacional sobre los Derechos de las personas con Discapacidad, en cuyo primer artículo incluye las deficiencias físicas, mentales, intelectuales o sensoriales a largo plazo que, al interactuar con diversas barreras, pueden impedir su participación plena y efectiva en la sociedad, en igualdad de condiciones con las demás" (CIPD, 2006, p. 4).

Por su parte, recién en el año 2008, el Estado Chileno ratifica la Convención Internacional sobre los Derechos de las Personas con Discapacidad y asume la obligación de hacer frente a los obstáculos creados socialmente, con el fin de promover y garantizar el pleno respeto de la dignidad y la igualdad de derechos de todas las personas, la autonomía e independencia de las personas con discapacidad en un contexto de igualdad de oportunidades con las demás, así como impulsar el fortalecimiento de la sociedad civil en temas de discapacidad mediante la creación de Políticas y Planes Públicos que faciliten la coordinación y aplicación de medidas transversales de forma participativa (PLANDISC, 2013). A pesar que se dicta en Chile la Ley 20.422 durante el año 2010, estableciendo normas sobre Igualdad de Oportunidades e Inclusión Social de las Personas con Discapacidad, esto no es suficiente si no se acompaña de una política pública, es decir, un programa que el gobierno desarrolle en función de un problema o situación determinada (Ruiz y Cadenas, 2015). Entonces, en enero del año 2013 se publica el Plan Nacional de Acción para la Inclusión Social de las Personas con Discapacidad 2013-2020, desarrollado en conjunto con el Proyecto Unión Europea, la Agencia de Cooperación 
Internacional de Chile y el Servicio Nacional de la Discapacidad (SENADIS), cuyo propósito es instalar en la gestión pública, acciones coherentes con los lineamientos planteados en las convenciones internacionales (PLANDISC, 2013, p. 6).

Dentro de los principales ámbitos de la Política Nacional de Discapacidad se encuentra la accesibilidad universal, citada como:

La condición que deben cumplir los entornos, procesos, bienes, productos y servicios, así como los objetos o instrumentos, herramientas y dispositivos, para ser comprensibles, utilizables y practicables por todas las personas en condiciones de seguridad y comodidad y de la forma más autónoma y natural posible (Roig, 2005, p. 20).

Para evaluar la accesibilidad universal, cabe mencionar dos dimensiones que están asociadas: la dimensión objetiva, caracterizada por un adecuado diseño y construcción, y la dimensión subjetiva, es decir, la percepción que tienen los posibles usuarios de la compatibilidad de ese entorno con sus características y con sus aspiraciones. Por lo tanto, una "buena accesibilidad" es aquella que pasa desapercibida a los usuarios. Esta "accesibilidad desapercibida” implica algo más que ofrecer una alternativa al peldaño de acceso, pues busca un diseño equivalente para todos, cómodo, estético y seguro. Se refiere a la "capacidad de aproximarse, acceder, usar y salir de todo espacio o recinto con independencia, facilidad y sin interrupciones" (Boudeguer y Squella, 2010, p. 12).

El Centro para el Diseño Universal de la Universidad de Carolina del Norte define siete principios básicos en los que se ha de basar el desarrollo de entornos bajo este concepto: Igualdad de uso (adecuado para todas las personas, independientemente de sus capacidades y habilidades); Flexibilidad (se acomoda a una amplia variedad de capacidades); Uso simple y funcional (intuitivo y fácil de entender independiente de la experiencia, conocimientos, habilidades o nivel de concentración del usuario); Información comprensible (intercambia información con el usuario, independiente de las condiciones ambientales o las capacidades sensoriales del mismo, por 
ejemplo: formas de información gráfica, verbal o táctil); Tolerancia al error (reduce al mínimo los peligros); Bajo esfuerzo físico (eficazmente usado en una posición neutral del cuerpo mientras utiliza el elemento, minimiza las acciones repetitivas y el esfuerzo físico sostenido) y Dimensiones apropiadas (los tamaños y espacios deben ser apropiados para el alcance, manipulación y uso por parte del usuario, independientemente de su tamaño, posición o movilidad).

También es importante señalar que la situación de discapacidad desde un punto de vista teórico ha ido cambiando a lo largo de la historia, por ende, sus aproximaciones y formas de intervención también se han visto afectadas. El II estudio de discapacidad del año 2015 en su construcción conceptual, da cuenta de una evolución desde la teoría de la normalización y la integración, hacia el modelo de derechos humanos y la inclusión.

La teoría de la normalización fue definida en el año 1959 por el Servicio Danés para el Retraso Mental como "la posibilidad de que los deficientes mentales lleven una existencia tan próxima a lo normal como sea posible”. En el año 1972, W. Wolfensberger amplía el concepto redefiniéndolo como:

La utilización de medios culturalmente normativos (familiares, técnicas valoradas, instrumentos, métodos, etc.) para permitir que las condiciones de vida de una persona (ingresos, vivienda, servicios de salud, etc.) sean, al menos, tan buenas como las de un ciudadano medio, para mejorar o apoyar en la mayor medida posible su conducta (habilidades, competencias, etc.), apariencia (vestido, aseo, etc.), experiencias (adaptación, sentimientos, etc.), estatus y reputación (etiquetas, actitudes, etc.) (García y Sánchez, 2004, p. 3).

La teoría de la normalización, que significó un gran aporte para la definición del modelo social, tuvo como principal consecuencia la generación del Informe Warnock (1978), elaborado por el sistema educativo inglés, donde se plantea por primera vez el principio de integración. En este documento se manifiesta que la integración propugna la escolarización conjunta de alumnos 'normales' 
y con discapacidades, abogando por la inserción de la educación especial en el marco educativo ordinario, "prestando siempre la atención adecuada y necesaria de cada alumno, defendiendo la atención a las características y necesidades de forma individualizada, adaptando los programas, métodos y recursos de cada caso concreto en el marco de la educación regular" (Rubio, 2009, p. 56). La integración es una cuestión de localización de las personas con discapacidad en sus entornos, donde tienen que adaptarse a la organización y estructuras existentes, considerando que el problema está en el sujeto y que éste requiere condiciones especiales y adaptaciones del sistema. En este modelo, se inserta a la persona 'distinta' al grupo normalizado y, como excepción, se diseñan programas específicos para atender sus diferencias.

A partir de la Conferencia Mundial sobre Necesidades Educativas Especiales de la Organización de las Naciones Unidas para la Educación, efectuada el año 1994, se inicia el proceso para establecer la inclusión como nuevo principio asociado a la discapacidad, el que, al igual que la integración, surge dentro del ámbito educacional y luego es llevado al plano social. Según la publicación del año 2005 en relación al Seminario Internacional "inclusión Social, Discapacidad y Políticas Públicas” (UNICEF y Ministerio de Educación de Chile), las PsD tienen derecho a la igualdad de oportunidades y a la plena inserción social. Esto implica conseguir un cambio cultural para que esta población sea mirada desde sus potencialidades y no se encasille sólo en su déficit. La discapacidad entendida en su contexto social, es una experiencia de diferencia y también de exclusión y opresión por la falta de comprensión de la sociedad. Promover una vida más justa y equitativa es el desafío actual, a través del enfoque de derechos en las políticas de públicas y la provisión de servicios médicos y sociales.

\section{Contexto institucional del estudio: modelo de salud familiar y comunitaria}

La historia de nuestro país ha mostrado la importancia del rol de la Atención Primaria de Salud, en su capacidad de hacer frente a 
eventos políticos y sociales, tales como la Epidemia del Cólera en el siglo XIX, la creación del Servicio Nacional de Salud (1952), municipalización de la salud primaria (década del 1980) y la creación de los Centros de Salud Familiar (1997). Ello impulsó al sistema de salud, bajo el nuevo modelo de atención integral, hacia una cobertura universal y de indicadores sanitarios similares a países desarrollados. Este modelo persigue la propuesta de la Organización Mundial de la Salud (OMS) que, desde el año 2008, promueve cambios en beneficio de la cobertura y atención centrada en la persona, su familia y la comunidad para avanzar en la protección de la salud, con mayor equidad y justicia social.

De este modo, a fines de la década de los noventa, el Ministerio de Salud de Chile impulsa la implementación de un nuevo modelo de atención para el nivel primario denominado Salud Familiar y Comunitaria. Esto significó un cambio de paradigma desde un enfoque de atención esencialmente biomédico hacia el biopsicosocial. Este nuevo enfoque entiende que la atención sanitaria debe ser un proceso continuo que se centra en el cuidado de la persona y sus familias, preocupándose de la prevención de la enfermedad y/o sus complicaciones a través del autocuidado y promoción de estilos de vida saludables para mejorar la condición de salud.

Por lo tanto, el modelo de atención integral es entendido como:

El conjunto de acciones que promueven y facilitan la atención eficiente, eficaz y oportuna, que se dirige más que al paciente o la enfermedad como hechos aislados, a las personas consideradas en su integralidad física y mental, como seres sociales pertenecientes a distintas familias y comunidades, que están en permanente proceso de integración y adaptación a su medio ambiente físico, social y cultural (Subsecretaría de Redes Asistenciales, División Atención Primaria, 2016, p. 18).

Es importante mencionar que la Salud Familiar y Comunitaria se desarrolla esencialmente en el contexto de la APS y constituye su elemento nuclear de intervención. En este sentido, es necesario entender que la APS es el eje central del modelo biopsicosocial 
implementado en los distintos Centros de Salud Familiar (CESFAM) que se encuentran desplegados en el territorio nacional para ofrecer una atención equitativa y centrada en la persona.

El Ministerio de Salud de Chile define su modelo de atención como un sistema basado en la Atención Primaria administrada por los municipios, desde donde se establecen los principios que orientan el quehacer de los equipos en la red asistencial, comenzando con la anticipación al daño hasta la rehabilitación, incluyendo otras necesidades de salud en el espacio de la familia y la comunidad (MINSAL, 2016). Por su parte, los administradores municipales describen al sistema comunal de salud, como:

La unidad básica organizativa de la APS, que forma parte de una entidad global plenamente articulada con el Sistema Nacional de Salud, el cual constituye el punto focal de planificación estratégica y gestión local de la Atención Primaria. Siempre bajo la conducción rectora y normativa del nivel central (Ministerio), donde se formulan las políticas globales y se definen los requisitos técnicos y administrativos que requiere la prestación de servicios en el nivel local. En este marco, se establece como misión de la salud municipal: Asegurar el desarrollo y funcionamiento de un sistema de atención primaria de salud integral, resolutivo y oportuno, mediante el diseño, instalación, monitoreo y evaluación de un plan de salud comunal y del uso eficiente y racional de los recursos para su implementación (MINSAL, 2016).

Por último, es importante señalar que, como la Atención Primaria es el eje del modelo biopsicosocial y la base del sistema de salud chileno, adquiere mayor relevancia la temática de "accesibilidad" en los centros de salud familiar para las personas en situación de discapacidad, en especial, cuando ésta se manifiesta en sectores de alta vulnerabilidad social e incide en su calidad de vida.

\section{Antecedentes metodológicos}

La metodología de esta investigación se sustenta en el paradigma de la "complejidad", el cual está precedido por las bases de la teoría ge- 
neral de sistemas, con el fin de visualizar la realidad como un todo, complejizando a los sistemas, tanto en una realidad analítica como dialéctica. El paradigma de la "complejidad" aglutina a científicos de diversos campos de conocimiento que insisten en la conveniencia de adoptar nuevos modelos teóricos, metodológicos y, por ende, una nueva epistemología. Ésta permite a la comunidad científica elaborar teorías más ajustadas de la realidad, que posibiliten al mismo tiempo, diseñar y poner en práctica modelos de intervención social, sanitaria, educativa, política, económica, ambiental, cultural, entre otras. Este planteamiento ayuda a encontrar nuevos enfoques epistemológicos para el desarrollo de tácticas o estrategias que permitan acciones individuales y colectivas, afectando indistintamente a las ciencias duras como a las humanistas.

La complejidad trasciende una visión multidisciplinaria, pues no es la suma de sus partes, ni tampoco un conjunto de disciplinas que pueden dar a conocer un fenómeno (Morin, 2008). Es en este contexto que el grupo de investigación consideró relevante abordar dicho fenómeno social, no tan sólo desde un aspecto cualitativo, sino también, cuantitativo. Ambos métodos permiten integrar datos e información relevante para una comprensión global de la situación social de las PsD, tanto desde la objetividad de los datos, como la subjetividad de la observación del fenómeno investigado.

El método utilizado en esta investigación es denominado:

Método mixto, el cual representa un conjunto de procesos sistemáticos, empíricos y críticos de investigación. A su vez, implican la recolección y el análisis de datos cuantitativos y cualitativos, así como su integración y discusión conjunta, para realizar inferencias respecto de la información recabada y lograr un mayor entendimiento del fenómeno estudiado" (Hernández y Baptista, 1998, p. 489).

Entonces, lo cualitativo entregaría la perspectiva de las personas a estudiar, generando un conocimiento más asertivo y representativo, donde el relato de experiencias e historias de vida, permite comprender la realidad en la cual están insertas las PsD. Por otra parte, 
el modelo cuantitativo trabajaría con datos más dirigidos, que miden un conjunto de variables, permitiendo describir a la población observada. En el contexto de un estudio mixto, el método utilizado fue el denominado diseño de Triangulación, cuyo propósito es combinar las fortalezas de ambas metodologías para obtener datos complementarios acerca de un mismo problema de investigación. El investigador deseaba comparar y contrastar los datos originados por estas distintas metodologías. Específicamente el diseño de Triangulación fue transformativo concurrente, pues se recolectaron datos tanto cualitativos como cuantitativos en un mismo momento. La finalidad de ello, según Creswell (1998), es lograr que la información cuantitativa y cualitativa dialogue en su análisis, logrando la confluencia de la información. En este sentido, los investigadores realizaron el estudio en una sola etapa donde simultáneamente se recolectó, procesó y analizó la información obtenida.

El equipo de investigación optó por utilizar el método mixto, pues se carecía de otros estudios previos en la temática de discapacidad a nivel institucional, comunal y regional; y dada la complejidad socio-sanitaria de las $\mathrm{PsD}$, dicho método permitiría una comprensión y descripción más profunda del fenómeno a nivel local.

Este estudio se sitúa desde el diseño investigativo no experimental, es decir, es un método donde no existe manipulación de variables de manera intencionada por parte de el o los investigadores. Se trata de investigaciones donde las variables siguen su curso natural para ver sus efectos y/o consecuencias sobre otras variables relacionadas (Kerlinger \& Lee, 2002). De ahí se deduce que no interfieren estímulos anexos sobre los individuos y se obtiene la información en su contexto natural. Esto da mayor confiabilidad a los datos e información para su posterior análisis, por ello, el grupo de investigación se planteó el propósito de conseguir la información de manera natural, sin generar situaciones ajenas a lo cotidiano.

El estudio fue de tipo exploratorio con alcance descriptivo, pues no existen estudios previos de esta población objetivo en el CESFAM, por ende, por primera vez existía un acercamiento científi- 
co respecto de las principales categorías que interesa profundizar (cómo es y cómo se manifiesta el fenómeno de interés). Esta descripción puede ser más o menos profunda, pero en cualquier caso, "se basa en la medición de uno o más atributos del fenómeno descrito" (Hernández y Baptista, 1998, p. 61), de manera de permitir descubrir el significado dado por las PsD, a la accesibilidad en el CESFAM donde son atendidos.

Las técnicas de recolección de información fueron la entrevista grupal a informantes claves. Desde esta metodología prevalece la conversación cara a cara, donde la persona que participa en la investigación tiene la posibilidad de opinar libremente, e incluso, formular preguntas y reflexionar en torno al tema que le ha propuesto el investigador. Su fundamento se basa en que la realidad social está construida sobre los marcos de referencias de los actores, siendo el paradigma interpretativo el que permite comprender las conductas verbales y físicas de las personas, capturando el significado que ellas les dan a los acontecimientos. En suma, interesa comprender la conducta humana desde el propio marco de referencia de quien actúa. Para el enfoque cuantitativo se utilizó un cuestionario, que se define como un conjunto de preguntas respecto a una o más variables a medir. El cuestionario fue estandarizado. Las posibilidades de respuestas fueron dicotómicas y de selección múltiple. La elaboración del instrumento consideró un lenguaje adecuado, un orden establecido, las condiciones del contexto y la estética del instrumento, contando con una introducción y un orden lógico.

Uno de los criterios de validez considerado en este estudio fue la "saturación de la información". Ésta consiste en "reunir las pruebas y evidencias suficientes para garantizar la credibilidad de la investigación... Permite tener la flexibilidad de determinar el momento en el cual la información entregada se repite hasta cubrir la categoría de análisis" (Pérez, 2004, p. 67). Otro criterio utilizado fue la triangulación de técnicas, lo que se logró a partir de las entrevistas grupales y la recolección de los datos cuantitativos. Esto permitió un conocimiento profundo respecto de cada una de las categorías propuestas. Y por último, fue considerado el criterio ético vinculado al 
consentimiento informado, el cual procura la voluntariedad de la participación y el resguardo de la confidencialidad de la información obtenida. Finalmente, el acercamiento al campo fue a través de contactos personalizados para explicar el objetivo de la entrevista y el cuestionario. Las entrevistas se aplicaron en el salón de reuniones del CESFAM y los cuestionarios en las atenciones y visitas domiciliarias realizadas por el equipo de profesionales.

\section{Principales resultados}

Para el análisis de los resultados de esta investigación se trabajó según las características de los estudios con metodología mixta. Desde la dimensión cualitativa se empleó el análisis de contenido del esquema dado por Taylor y Bogdan (1992), el cual consiste en un primer momento, en la identificación de temas, conceptos y proposiciones (proceso denominado “Categorización”) y en un segundo momento, cuando los datos ya han sido recogidos, se asigna un valor a los textos (proceso denominado "Codificación"). En este sentido, el equipo investigador trató de relativizar sus descubrimientos, de manera de comprender los datos en el contexto en el cual fueron recogidos (proceso denominado "Interpretación”). Desde la dimensión cuantitativa se procesaron los datos a través de la estadística inferencial descriptiva, cuyo objetivo fue recoger, clasificar y analizar las características de un conjunto de elementos, deduciendo conclusiones sobre su estructura y composición, es decir, este tipo de análisis tuvo como objetivo realizar estimaciones a partir de los resultados obtenidos, permitiendo obtener visiones de los usuarios del centro de salud en cuanto a las barreras y fortalezas en la accesibilidad al CESFAM.

En el contexto de lo anterior, antes de iniciar el análisis de la información, el equipo de investigación asumió que la política social busca, dentro de sus orientaciones estratégicas, "promover y garantizar el pleno respeto de la dignidad y la igualdad de derechos de todas las personas con discapacidad". Sin embargo a nivel local, esto se invisibiliza, dado que existen una serie de factores, tanto institucionales como del entorno, que están afectando la realidad de estas personas. 
Es importante señalar que la "Accesibilidad" es la categoría principal del estudio y es definida como la interacción multidireccional entre la persona y el contexto socio-ambiental, condición que deben cumplir los entornos, procesos, bienes y servicios para que todas las personas puedan, sin dificultades, acceder a un conjunto de prestaciones que en este caso son ofrecidas por el Estado, con la intención de aportar al mejoramiento de la calidad de vida de las PsD.

Es por ello que a continuación se analizan un conjunto de dimensiones que son parte de la accesibilidad universal y que actúan interrelacionadamente en la igualdad y en el acceso al desplazamiento, infraestructura, transporte, información y atención oportuna, como aspectos del medio social que están generando mayor vulnerabilidad en las PsD.

En relación al desplazamiento, se puede señalar que la discapacidad física es la que se presenta en mayor porcentaje en los usuarios inscritos, con un 64\%. Este dato es relevante dado que nos permite conocer el tipo de discapacidad que mayor demanda genera en horas de atención para el centro de salud. Demanda que debe ser uno de los focos prioritarios en la prestación de servicios.

\section{Gráfico $\mathrm{N}^{\circ} 1$}

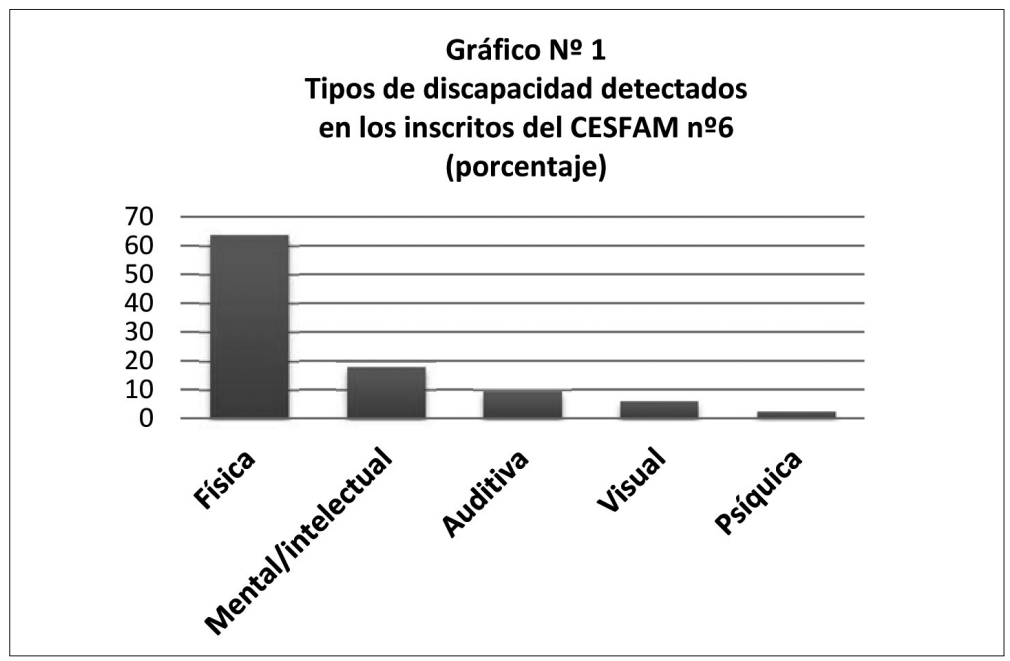

Fuente: Elaboración propia. 
El gráfico $n^{\circ} 1$ muestra que la discapacidad física es la más frecuente en los usuarios inscritos en el CESFAM n ${ }^{\circ} 6$ con un $64 \%$, seguido de la discapacidad Mental / Intelectual con un 18\%. La discapacidad sensorial representada alrededor del $10 \%$ por la Auditiva, seguida de la discapacidad Visual que alcanza un 6\%. La discapacidad que menos representación tiene, es la de tipo psíquico con un $2 \%$.

Ahora bien, considerando lo anterior y a partir de las entrevistas grupales, se puede señalar que el desplazamiento es entendido como la capacidad de aproximarse, usar y salir de todo espacio, con independencia y facilidad; se trata de lineamientos estratégicos declarados en la política nacional de la discapacidad que constituyen el marco regulatorio para las nuevas infraestructuras en nuestro país. La infraestructura del CESFAM nº data del año 2007, por lo que cuenta con las normas establecidas en el Programa Médico Arquitectónico (PMA). Los participantes identifican adecuaciones acordes a sus necesidades, tales como rampas o puertas de acceso a oficinas, lo que es percibido por los entrevistados de manera positiva (gráfico 2).

Gráfico $N^{\circ} 2$

\section{Gráfico №2 \\ ¿Cómo evalúa el desplazamiento en el CESFAM no6 de Rancagua?}

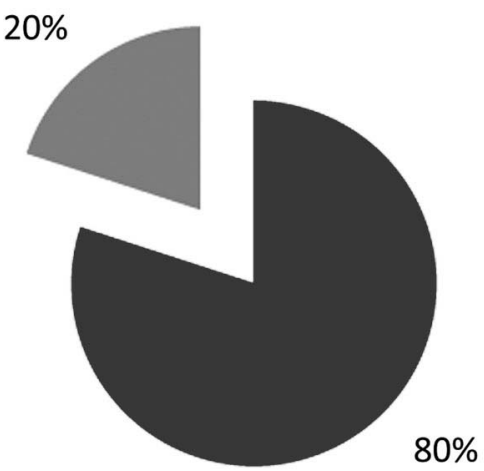

- Fácil

Difícil

Fuente: Elaboración propia 
Este gráfico nos indica que el $80 \%$ de los encuestados considera "fácil" el desplazamiento dentro del recinto ya que lo caracteriza como "amplio" y valoran el hecho de ser una planta única ("no tiene escaleras"). Sin embargo, el 20\% restante señala la dificultad de su desplazamiento al "requerir de un acompañante", "usar muletas" y observa un "deterioro en la rampla".

["La entrada está bien, se puede entrar con facilidad está todo bien, muy bien implementado”. (EG2/AD)]

[“Sí, las puertas y la rampla están bien.” (EG2/AI)]

Los relatos de los entrevistados dan cuenta de que las actuales instalaciones del CESFAM cumplen con los requisitos básicos para el desplazamiento de personas con discapacidad física, situación que permite una entrega más expedita de los servicios que el centro ofrece al facilitar el desplazamiento a las distintas instalaciones existentes. No obstante, el desplazamiento también depende de otros factores externos que pueden afectar la accesibilidad al centro de salud, por ejemplo, la existencia de cuidadores, de ayudas técnicas adaptadas a sus necesidades específicas y el correcto uso de la infraestructura. Éstas son dimensiones relevantes en este estudio, dado que el desplazamiento de personas con discapacidad no sólo implica un proyecto arquitectónico inclusivo, sino también, personas con una red social que facilite y apoye su condición, de tal manera que éstas colaboren activamente con un entorno social facilitador, más aún en la discapacidad de tipo intelectual, donde el cuidador asume un rol protagónico ineludible en la conexión entre la PsD y el centro de salud.

["Yo quisiera decir que yo en silla de rueda es un suplicio, porque mi hijo tiene deficiencia mental y él es mi chofer”. $(\mathrm{EG} 2 / \mathrm{AD})]$

["Dependo de una muleta y de una persona que esté a mi lado”. (EG1/AD)]

["Mi mamá falleció hace dos meses y ella me andaba trayendo para todos lados con mi silla de rueda”. (EG2/AD)] 
De acuerdo a lo anterior y desde la dimensión objetiva, el CESFAM cumple con las condiciones para que las personas puedan desplazarse sin mayores dificultades al interior del recinto, sin embargo, cuando se realiza el análisis en función del tipo de discapacidad, se advierte la importancia del apoyo de quienes hoy asumen un rol de cuidadores, pues la red social es fundamental para disminuir la vulnerabilidad de las PsD.

En esta dimensión, la política social realiza el esfuerzo de cumplir con los estándares para que la accesibilidad en estos recintos sea universal, pero también el esfuerzo se debe concentrar en aquellos factores ambientales que permitan facilitar y disminuir la vulnerabilidad social de estas personas, por lo tanto, las redes familiares son fundamentales para apoyar los procesos de integración, tanto en el desarrollo de sus habilidades y/o autonomía, como en hacer valer sus derechos en salud.

En este sentido, la necesidad de contar con una infraestructura adecuada ha sido relevante para garantizar la accesibilidad universal (un adecuado diseño en construcción equivalente para todos, cómodo, estético y seguro), la normativa señala que los espacios deben ser apropiados para el alcance, manipulación y uso por parte del usuario, aspectos que influyen en la inclusión al momento de entregar una atención médica.

Es en este contexto que el CESFAM n ${ }^{\circ} 6$ cumple con cada uno de los requisitos técnicos establecidos en la normativa, situación que se transforma en una oportunidad para la institución, pues contar con una infraestructura acorde a las necesidades de las PsD, aporta en la calidad de la atención que se les brinda. Por otro lado, se logra que las personas se desplacen sin dificultades al interior de las instalaciones del establecimiento. Así lo muestran las entrevistas grupales:

[“La entrada está bien, se puede entrar con facilidad... está todo bien... muy bien implementado". (EG2/AI)]

[“La infraestructura la encuentro buena" (EG1/AI)] 
Por lo tanto, la infraestructura cumple con las expectativas que la comunidad usuaria le exige a un centro de atención primaria público, entre las cuales se valora su luminosidad, tamaño, comodidad y seguridad en las instalaciones (GRÁFICO 3).

Gráfico 3

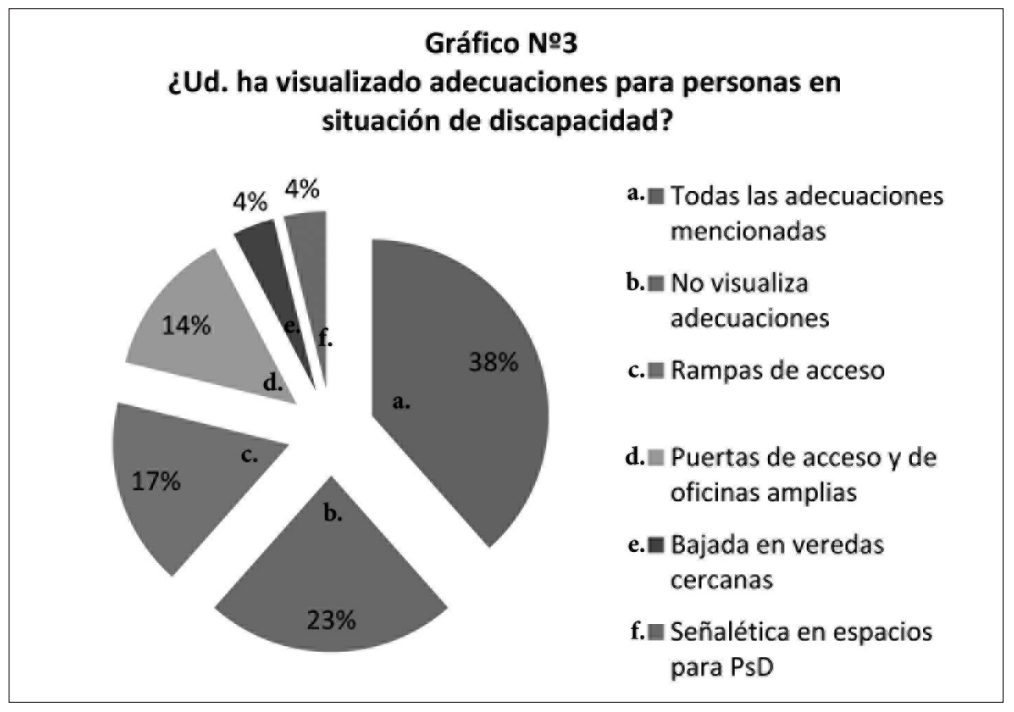

Fuente: Elaboración propia.

Finalmente, en conocimiento que la infraestructura data del año 2007 y cumple con las normas establecidas en el Programa Médico Arquitectónico (PMA), los encuestados en su mayoría identifican adecuaciones acordes a sus necesidades, tales como rampas o amplitud en las puertas de acceso a las oficinas. Sin embargo, un $23 \%$ de la muestra no identifica adecuaciones y un dato destacable es el bajo porcentaje de señalética que las PsD identifican dentro del recinto; todos aspectos relevantes a considerar en la elaboración de políticas locales inclusivas al interior del CESFAM.

Otra dimensión fundamental en la accesibilidad universal se refiere al transporte, que se reconoce como uno de los problemas fundamentales que imposibilita el acceso a diferentes servicios (centros comerciales, espacios de recreación, recintos de salud, etc.). Esto impacta tanto en el bienestar emocional de las PsD, como en 
sus propias familias. Cabe mencionar que si bien la discapacidad se da principalmente en contextos de pobreza, en "Chile la discapacidad impacta en la vulnerabilidad de las personas en todos los niveles socioeconómicos, pues los accesos a la salud, educación y trabajo, entre otros, no se relacionan sólo con la falta de recursos monetarios, sino también a las barreras actitudinales y culturales, junto con el desconocimiento de la discapacidad" (Cabieses y otros, 2016, p. 398).

Ante la situación vivida por las PsD, el Estado promulga la ley 20.422, que establece normas sobre Igualdad de Oportunidades e Inclusión Social de las personas con discapacidad. El desarrollo de la accesibilidad también está enfocado al uso de los medios de transporte en igualdad de condiciones, es decir, las políticas de transporte público deben permitir el acceso igualitario, independiente de la condición de la persona, a un servicio que cuente con las garantías mínimas para el traslado desde sus lugares de residencia hacia los respectivos centro de atención.

Si bien los marcos regulatorios están establecidos por la autoridad respectiva, existen componentes culturales que obstaculizan el uso de los medios de transporte en igualdad de condiciones, pues el hecho de vivir con alguna discapacidad, conlleva al trato discriminatorio de las $\mathrm{PsD}$ por parte de los conductores, al rechazar transportarlos, y del público en general, al no ceder el espacio preferencial.

["Los choferes a ti con muletas no te paran y menos aún con silla de ruedas. La gente de las micros es descortés cuando paran... no te ayudan" (EG1/AT)]

["Yo he estado desde las 6 am hasta la 8 am esperando que me lleve un colectivo”. (EG2/AT)]

["La micro primera no me paró y nadie se paró a darme el asiento”. (EG2/AT)]

Los participantes señalan, que además de trasladarse al CESFAM $\mathrm{N}^{\circ} 6$ por sus propios medios, también escogen la locomoción co- 
lectiva como primera preferencia (gráfico 4). Se puede señalar que el acceso al centro de salud se ve perjudicado debido a la percepción negativa que los usuarios relatan al usar transporte público. Éste no cuenta con adecuaciones necesarias para transportar a PsD. A pesar de lo descrito, la locomoción colectiva representa el único medio disponible para el traslado de estas personas.

Gráfico $\mathrm{N}^{\circ} 4$

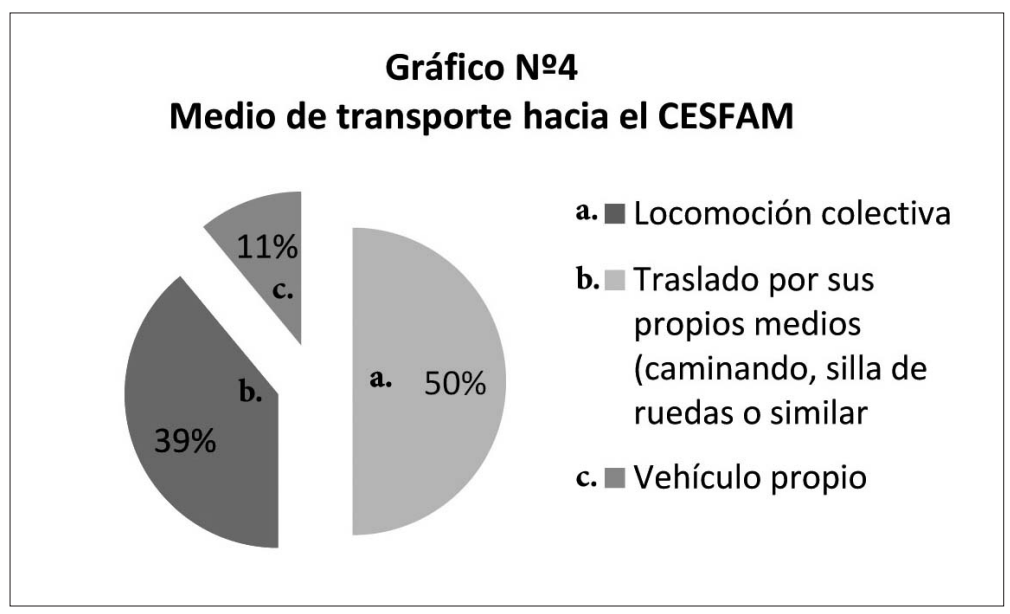

Fuente: Elaboración propia

El gráfico $\mathrm{n}^{\circ} 4$ muestra que la mitad de los participantes se traslada al CESFAM N ${ }^{\circ} 6$ de Rancagua en locomoción colectiva y la segunda mayoría, que asciende a un $40 \%$, lo hace por sus propios medios. Tan sólo un $11 \%$ cuenta con vehículo propio. En este sentido las "discriminaciones son fenómenos humanos indisociables de las circunstancias determinadas por el tiempo y el espacio. Además, se desarrollan en un complejo sistema socio-cultural caracterizado por prácticas, representaciones y narrativas contra grupos de personas" (Machin, 2013, p. 87).

La dinámica y lógica, interrelacionadas entre sí detrás del fenómeno de la discriminación, abre un campo de debate entre las distintas áreas que influyen en la perpetuación de ésta, como por ejemplo: el costo financiero para el conductor cuando transporta una PsD (economía), la sanción legal (jurídica), la sanción moral 
(cultural), la participación social en la elaboración de las políticas públicas (política) o la jerarquización de beneficios (poder y resistencia). Es decir, la dimensión simbólica detrás de las PsD, es producto de un constructo colectivo basado en la historia de una sociedad y se despliega acorde al contexto donde se instale para conferir determinados códigos de interpretación, los cuales recaen finalmente sobre el grupo "discriminado" y que, de no recapacitar en torno a los daños causados, se traspasan de generación en generación. Es por ello que sensibilizar en esta problemática, crea una condición de posibilidad para dar el primer paso hacia una sociedad inclusiva en todos los ámbitos.

Otro de los aspectos identificados por las PsD como relevantes y que influyen en la accesibilidad universal, es la falta de acceso a la información, pues el desconocimiento de las redes institucionales disponibles, dificulta mejorar la igualdad de oportunidades de las PsD dentro de la sociedad chilena (gráfico 5). Esto aumenta la percepción de desamparo y de falta de acceso a un conjunto de beneficios.

Tanto la institucionalidad pública, como de la sociedad civil, contribuyen al aumento de la vulnerabilidad social de las PsD. Esto se recoge en la entrevista grupal:

["También tenemos algunas trabas de la municipalidad, tenemos que andar pidiendo ayuda, como pedir limosna para que nos ayuden... yo tengo conocimiento de haber estado en otras instituciones. Yo he estado en varias que me han defraudado". (EG1/AI)]

["Lamentablemente en nuestro país, la ley de discriminación es para los puros gays, y a nosotros... ¿Quién nos defiende cuando nos tratan de "locos", cuando nos tratan de "la coja"?" (EG1/AI)]

["Para nosotros, las personas que somos discapacitadas, todas las cosas que haga el país a nivel nacional a nosotros no nos sirven" (EG2/AI)] 
Complementa la información anterior el gráfico n5, ya que en relación a las instituciones dedicadas al tema de la discapacidad, se observó un desconocimiento generalizado de los servicios que se brindan a las PsD. Las instituciones más conocidas son la fundación Teletón y el Servicio Nacional de Discapacidad (SENADIS), por el contrario, la Oficina de Subsidios y la de Intermediación Laboral, son prácticamente desconocidas.

Gráfico $\mathrm{N}^{\circ} 5$

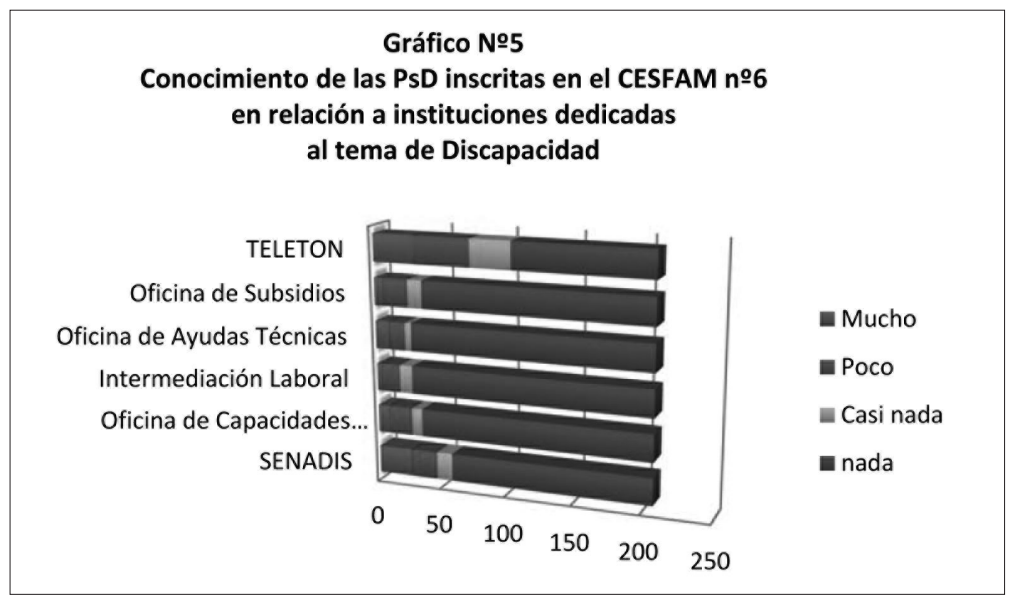

Fuente: Elaboración propia

La falta de información perjudica la accesibilidad de las PsD a los distintos servicios que ofrecen las instituciones, públicas y privadas, aumentando su descontento y vulnerabilidad. De acuerdo a lo anterior se puede señalar que si bien el CESFAM es el último eslabón en el área de salud representativo del Estado a nivel local, es el primero donde las personas acuden en búsqueda de una solución a su problemática sanitaria, por ende, fortalecer estos espacios es fundamental para articular una red intra-estatal que permita que el conjunto de beneficios logre llegar con mayor accesibilidad a las PsD.

La modernización del Estado plantea el "desafío de democratizar la sociedad chilena, en cuanto a un Estado ágil, activo al servicio de la ciudadanía" (...) "donde sus políticas sociales permitan llegar a aquellos sectores más desprotegidos de nuestro país" (...) "las políti- 
cas públicas se implementan, pero la información de sus beneficios no llega a este grupo específico de la población" (Torres, 2009, p. 10). Todos estos aspectos tensionan la relación entre el equipo de salud y los usuarios, ignorando las condiciones y necesidades especiales que se necesitan para abordar los problemas desde un enfoque integral.

Por último, siendo los CESFAM el acceso al Sistema Público de Salud, asume las necesidades expresadas por este grupo de personas usuarias como un desafío para la gestión y distribución de los escasos recursos con que se cuenta. Por ello, la atención oportuna se dificulta no sólo al ser considerados un grupo prioritario por su condición de discapacidad, sino que:

Influyen además otros factores que son propios de las falencias del modelo de Salud Familiar en Chile: tiempo limitado de atención a los pacientes, rotación de médicos tratantes, entre otros aspectos que afectan un adecuado seguimiento de la salud de las personas en situación de discapacidad (Cabieses y otros, 2016, p. 400).

Esta situación de carácter estructural, donde las instituciones deben ajustarse y adaptarse a la funcionalidad, tanto de la política social como de la realidad local, conflictúa la relación entre comunidad y equipo de salud. Esto se confirma con lo expuesto en la entrevista grupal, ya que los participantes solicitan un trato preferencial acorde a los problemas de accesibilidad detectados en el CESFAM:

["No veo que ellos te estén directamente ayudando a conseguir lo que necesitamos con urgencia”. (EG2/AO)]

["No tuvo la humanidad de darme una hora. Lamentablemente tuve que optar por acudir a un superior, porque como se dice, no se ponen en el lugar. Porque la calle está muy mala, entonces, a nosotros nos exigen lo mismo: levantarse a las 6 de la mañana para sacar un número. Creen que uno hace show con la muleta" (EG2/AO)]

["Yo vengo a las 4 de la mañana a sacar número, llego al mesón y les dan preferencia a otras personas" (EG1/AO)] 
["Yo aquí, no sé si es una idea loca, pero no se puede solicitar una atención especial para los enfermos crónicos y los que tengan discapacidad" (EG1/AO)]

En el gráfico $n^{\circ} 6$ se indica que casi un tercio de la muestra no menciona problemas dentro del CESFAM estudiado, sin embargo, el problema más relevante lo encabeza, con un $35 \%$, la falta de información y con un 30\%, la falta de horas de atención; el 12\% relata sentir maltrato y discriminación; y dentro de los problemas menos comunes, estuvo la adecuación de la infraestructura y el acceso.

Gráfico $\mathrm{N}^{\circ} 6$

\section{Gráfico №6 \\ Problemas en el CESFAM $n \div 6$ de Rancagua detectados por PsD (porcentaje)}

Adecuación o acceso Maltrato/discriminación Falta de horas de atención Falta de información Ninguno

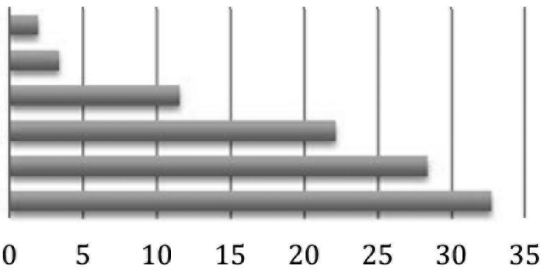

Fuente: Elaboración propia

En relación a la falta de atención expresada por los participantes, se requerirá, por una parte, un fuerte trabajo educativo con la comunidad usuaria y por otra parte, sensibilizar al equipo y plasmar dentro de la organización, la protección de la salud como un derecho fundamental que comienza con garantizar la oportunidad en el acceso.

De acuerdo a lo anterior, la organización del CESFAM, al ser el primer contacto de las PsD con el Sistema de Salud, debe exigir al equipo de profesionales actuar como un ente articulador dentro de la red institucional dedicada a la discapacidad, con el fin de aumentar el nivel de información que la comunidad discapacitada necesita para el pleno ejercicio de sus derechos. 


\section{Aportes a la construcción de políticas locales inclusivas}

La OMS (2010) define los determinantes sociales como las condiciones sociales en las cuales viven y trabajan las personas y son las que impactan en la salud, es decir, la salud se ve afectada por condiciones sociales más amplias: empleo, trabajo, condiciones sanitarias y ambientales, nivel socioeconómico, nivel de educación alcanzado por la persona, si es hombre o mujer, si pertenece a un pueblo indígena o no, si vive en la ciudad o en áreas rurales. Por ejemplo, la influencia de la posición económica de la persona sobre su salud no es directa, sino producto del actuar de una serie de factores como las condiciones materiales, la calidad de la vivienda y circunstancias psicosociales, incluyendo el estrés y los comportamientos, como el hábito de fumar o de alimentarse mal. El enfoque de determinantes sociales permite:

Fortalecer una visión colectiva y social de la promoción de la salud, además, es una invitación a mirar la salud de las personas y de las sociedades a través de la óptica más social y estructural del contexto donde éstas viven y se desarrollan durante los distintos ciclos de vida (López y Covarrubias, 2010, p. 12).

La situación de discapacidad es una:

Condición de vulnerabilidad que surge principalmente en poblaciones con vulnerabilidades sociales previas. En Chile existe una gran variabilidad en los datos y estadísticas respecto de la prevalencia y características de la población en situación de discapacidad, lo que dificulta la comparación de datos y la identificación de áreas en que se necesitan mayores intervenciones o de carácter más urgente. Por esto, resulta necesario realizar estudios específicos que permitan el diagnóstico, caracterización y definición de necesidades particulares de salud de esta población (Cabieses y otros, 2016, p. 404).

Es en ese contexto que este estudio ha permitido aportar algunas orientaciones estratégicas, las que en ningún momento pretenden ser un recetario de acciones a ejecutar en los espacios locales, pues 
entenderlo así sería un error epistemológico que nos daría una visión reduccionista de la realidad social.

Estas propuestas permiten fortalecer los procesos democráticos a nivel local, dado que se hace relevante hoy en día co-construir espacios de desarrollo en conjunto con los diversos actores del territorio. Los lineamientos que se proponen a continuación pueden ser implementados por diferentes estamentos públicos o privados. Para ello es necesario considerar la flexibilidad y la heterogeneidad del territorio, respetando la diversidad de actores y la cultura organizacional de cada institución.

Desde el trabajo interdisciplinario es fundamental reflexionar a partir de cada campo profesional, pues toda acción que se ejecute en los territorios nos invita y nos genera la necesidad de poder cuestionarnos críticamente nuestras formas de intervención profesional. Esto implica reflexionar sobre nuestras prácticas sociales y no asumir irreflexivamente la ejecución de nuestro hacer profesional.

De acuerdo a lo anterior, se sugiere utilizar la "Planificación Estratégica”, como una metodología de trabajo capaz de dar respuesta a las tareas cotidianas, que de forma planificada, apunta a atender y resolver consensuadamente con los funcionarios los temas de diseño, gestión y priorización de proyectos. Esta instancia metodológica proporcionaría una apropiación del proceso de la gestión por parte de las autoridades, los funcionarios y los ciudadanos. La adopción de esta estrategia, como herramienta de trabajo, significaría un cambio de actitud, pues se buscaría reemplazar la atención de actividades puntuales y emergentes, por "acciones estratégicas encaminadas a generar un espiral de desarrollo y participación” (Torres, 2008, p. 15). La realidad de las PsD requiere del trabajo concertado del conjunto de actores que son parte del territorio, es decir, el trabajo colaborativo entre las organizaciones comunitarias, iglesias, ONG, escuelas, CESFAM, municipios, y otros, con la finalidad de recoger la diversidad de visiones en torno a la temática estudiada.

Es posible plantear que la intervención en la realidad de las PsD, puede articularse de acuerdo a las decisiones estratégicas que ela- 
bore el equipo ejecutor. En este sentido, los equipos interdisciplinarios se constituyen en agentes dinamizadores de los procesos sociales, por ende, cumplen un rol activo al interior del CESFAM, donde es necesario que cuenten con las herramientas básicas de trabajo que permitan captar los marcos interpretativos de las PsD con la intención de: a) Promover una política local que logre articular las diversas redes institucionales con el fin de abordar los factores que influyen en la calidad de vida de las PsD de manera integral, en el sentido de que aquellas dialoguen y prioricen acciones de manera mancomunada y que mejoren la focalización de las acciones dirigidas a las necesidades sentidas por la comunidad. b) Generar programas y proyectos sociales desde una lógica situacional que se enfoque no sólo en quienes actúen como cuidadores principales, sino en un conjunto de actores involucrados, fortaleciendo los factores protectores que colaboren en el cuidado de las PsD. c) Promover una relación colaborativa entre ciudadanía y CESFAM que permita construir un vínculo, donde ambos actores complementen sus roles, incorporando una visión más amplia de la gestión territorial en salud.

\section{Bibliografía}

Boisier, S. (1999). Teorías y metáforas sobre el desarrollo territorial. Publicación de las Naciones Unidas. Santiago de Chile.

Briones, G. (1998). Métodos y Técnicas de Investigación para las Ciencias Sociales. Editorial Trillas. México.

Boudeguer, Prett y Squella (2010). Manual de Accesibilidad Universal. Santiago, Chile. Recuperado el 20 de diciembre de 2016 de http://www.ciudadaccesible.cl/wpcontent/ uploads/2012/06/manual_accesibilidad_universall.pdf.

Características de los Derechos Humanos (2008). Recuperado el 8 de noviembre de 2015 de: www.derecho.guia2000.com.

Convención Internacional sobre los Derechos de las Personas con Discapacidad (2016). Recuperado el 15 de mayo de 2017 de: http://www.un.org/esa/socdev/enable/documents/tccconvs.pdf

Primer diagnóstico de Discapacidad de la comuna de Rancagua (2014). Informe obtenido de la Oficina de Capacidades Diferentes de la Ilustre Municipalidad de Rancagua. 
Guasch, D.; Hernández, J. (2010). La accesibilidad del entorno universitario y su percepción por parte de los estudiantes con discapacidad. Fundación ONCE. Universidad Politécnica de Cataluña. España. Recuperado el 15 de marzo del 2017 de: http://www2.congreso.gob.pe/sicr/cendocbib/con4_uibd. nsf/7503CE5CC0AEE1E105257C75005A200B/\$FILE/1_ pdfsam_OUD1_2010-cas.pdf

Hernández, R.; Fernández, C.; Baptista, P. (1998). Metodología de la Investigación Social, editorial Mc Graw - Hill. México.

Machin, J. (2013). Análisis conceptual de las discriminaciones, desde un enfoque antropológico. Ponencia para el Seminario para la formación de una ciudadanía a favor del derecho a la igualdad y la no discriminación en la Ciudad de México. Recuperado el 20 de diciembre de 2016 de: https:// es.scribd.com/doc/145766832/Discriminacion-un-enfoque-antropologico.

Naciones Unidas. Portal de los Derechos Humanos. Traducción Universidad de Salamanca (2013). Recuperado el 8 de noviembre de 2015 de: http://www.un.org/es/rights/overview/charter-hr.shtml www.un.org

Pérez, G. (1994). Investigación cualitativa: Retos e Interrogantes. Editorial La Muralla S.A. Madrid, España.

Política Nacional de Acción para la Inclusión Social de las Personas con Discapacidad 2013-2020. Servicio Nacional de la Discapacidad. Santiago, Chile. Recuperado el 20 de diciembre de 2016 de: http://www.ciudadaccesible.cl/wp-content/ uploads/2011/08/Politica-Nacional-para-la-Inclusion-Social-de-las-Personas-con-Discapacidad.pdf

Roig, R. (2005). El significado de la accesibilidad universal y su justificación en el marco normativo español. Informe realizado por un Equipo de Investigación del Instituto de Derechos Humanos "Bartolomé de las Casas", al amparo de un Convenio de Colaboración con el Ministerio de Trabajo y Asuntos Sociales, Secretaría de Estado de Servicios Sociales, Familia y Discapacidad, y la Universidad Carlos III de Madrid. Recuperado el 8 de noviembre del 2015 de: http:// www.cermi.es/sites/default/files/docs/colecciones/Elsignificadodelaaccesibilidaduniversalysujustific.pdf

Ruiz, D.; Cádenas, C. (2006-2007). ¿Qué es una política pública? IUS Revista Jurídica (18). Recuperado el 8 de noviembre de 2015 de http://taoppcomunicacion.weebly. com/uploads/6/9/3/8/6938815/ruiz_1\%C3\%93pez__c\%C3\%81denas_ayala_qu\%C3\%A9_es_un_pp.pdf 
Samaniego, P. (2006). Aproximación a las personas con discapacidad en América Latina. Ministerio de Trabajo y asuntos sociales. Editado por el Comité Español de Representantes de Personas con Discapacidad. Madrid, España. Recuperado el 3 de marzo de 2017 de: http://www.foal.es/sites/default/ files/docs/33_aproximacionrealidadpersonasdiscapacidadfoal1_0.pdf

Taylor, S.; Bogdan, R. (1992). Introducción a los Métodos Cualitativos de la Investigación. Editorial Paidós, Barcelona. España.

Torres, M. (2009). La Descentralización como Construcción de un Proyecto Político Democrático: Dimensión Fundamental en el Fortalecimiento de la Ciudadanía. Revista Rumbos TS (4) p: 10.

Torres, M. (2008). Las Administraciones Públicas en el contexto de la Modernización del Estado. Revista Divergencias (1) p: 15.

Valdés, E. (2013). Bioderecho, genética y derechos humanos. Análisis de los alcances jurídicos del Bioderecho europeo y su posible aplicación en Estados Unidos como fuente de derechos humanos de cuarta generación. Universitas, Revista de Filosofía, Derecho y Política (17) p. 139-163.

\section{Bibliografía web:}

http://www.senadis.gob.cl/pag/354/595/accesibilidad_universal_del_ entorno 